\title{
Uso de las sondas de alimentación en el paciente con demencia avanzada. Revisión sistemática
}

\author{
$M^{a}$ Luisa de la Rica Escuín ${ }^{a}$, Julia González Vaca ${ }^{a}$ \\ José Luis Oliver Carbonell ${ }^{a}$, Pedro Abizanda Soler ${ }^{a}$
}

\begin{abstract}
aServicio de Geriatría. Complejo Hospitalario Universitario de Albacete.

Correspondencia: Marisa de la Rica Escuín, C/ Octavio Cuartero $75.3^{\circ}$ A.02004 Albacete. Correo electrónico: marisadlrscn@hotmail.com

Recibido el 8 de abril de 2013 Aceptado para su publicación el 14 de mayo de 2013.
\end{abstract}

\begin{abstract}
RESUMEN
Introducción: La aparición de disfagia es marcador de la fase final de la demencia. Estudios hasta el 2000 ponían en entredicho los objetivos que justifican la colocación de sondas de alimentación, pero ésta sigue siendo una intervención frecuente en demencias avanzadas.

Objetivos: Responder a las siguientes cuestiones según la evidencia disponible: La alimentación por sonda PEG en demencias avanzadas: ¿previene aspiraciones?, ¿previene desnutrición?, ¿aumenta la supervivencia?, ¿se tratan de manera paliativa?

Metodología: Revisión bibliográfica. Estudios publicados 2000-2012. Inglés y castellano. Bases de datos: Pubmed, Google Scholar, Cochrane. Mesh: Dementia, Enteral nutrition, Nutritional support, Endoscopic gastrostomy, Tube feeding, Peg, Enteral feeding, Ethics, Quality of life, Palliative care, Dysphagia y Make decisions.

Resultados: 96 artículos cumplían criterios para incluir en este estudio. 24 españoles, 72 publicaciones extranjeras. Las sonda nasogástrica y sonda PEG no están indicadas en pacientes con riesgo de broncoaspiraciones; las indicadas por la ESPEN tampoco las previenen. La guía ESPEN no considera indicado usar sondas de alimentación en la última fase de la demencia; la pérdida de peso es consecuencia de la enfermedad. Estudios señalan factores que afectan negativamente a la supervivencia. La mortalidad en los primeros 30 días tras la colocación de la sonda es muy elevada. La demencia no es asumida como enfermedad terminal, provocando ambivalencia en la toma de decisiones al final de la vida. La formación en cuidados paliativos no oncológicos es muy escasa, hecho que favorece instauración de tratamientos desproporcionados.

Conclusiones: La alimentación enteral en la demencia avanzada sigue siendo una intervención frecuente, no hay evidencia que establezca que la alimentación por sondas prevengan las aspiraciones, ni se logre una mejora en el estado nutricional, prevenga la desnutrición o sus consecuencias. Tampoco se logra un aumento de la supervivencia, incluso hay estudios que plantean una disminución de la supervivencia. Estos pacientes no se benefician de cuidados paliativos, porque la demencia severa tradicionalmente no ha sido considerada como enfermedad terminal, aunque muchos estudios aseguran que se lograría una mejora de la
\end{abstract} calidad de vida en la fase final de la enfermedad.

\section{ABSTRACT}

Introduction: The onset of dysphagia is a sign of the final stage of dementia. Studies up to the year 2000 called into question the objectives which justify the insertion of a feeding tube, but this continues to be a frequent intervention in advanced dementias.

Objectives: To answer the following questions based on available evidence: Does feeding by PEG tubes in advanced dementia prevent aspiration? Does it prevent malnutrition? Does it increase survival rates? Are they treated palliatively?

Methodology: Bibliographic research. Studies published 2000-2012. English and Spanish. Databases: Pubmed, Google Scholar, Cochrane. MeSH: Dementia, Enteral nutrition, Nutritional support, Endoscopic gastrostomy, Tube feeding, PEG, Enteral feeding, Ethics, Quality of life, Palliative care, Dysphagia and Making decisions.

Results: 96 articles met criteria to be included in this study. 24 Spanish, 72 in international publications. Nasogastric and PEG tubes are not indicated in patients at risk of bronchoaspirations; neither do those indicated by ESPEN prevent them. The ESPEN guide does not consider the use of feeding tubes

to be suitable in the final stage of dementia; weight loss is a consequence of the disease. Studies suggest factors which affect survival negatively. Mortality in the first 30 days after insertion of the tube is very high. Dementia is not accepted as a terminal disease, causing ambivalence in decision taking at the end of life. Training in non-oncological palliative care is very limited, a fact which can increase the establishment of disproportionate treatments.

Conclusions: Enteral feeding in advanced dementia continues to be a frequent intervention; there is no evidence which establishes that tube feeding prevents aspirations, or that an improvement in nutritional status is achieved, or that it prevents malnutrition or its consequences. Neither are higher survival rates achieved; there are even studies which suggest lower survival rates. These patients are not benefited by palliative care because severe dementia has not traditionally been considered a terminal disease, despite the fact that many studies claim that a higher quality of life would be achieved in the final stage of the illness. 


\section{INTRODUCCIÓN}

La ingestión deficiente de alimentos es frecuente en las personas con demencia y puede ocurrir en los primeros estadios de la enfermedad, incluso antes de que se haya realizado un diagnóstico; la aparición de trastornos de la deglución constituye un claro marcador de la fase grave de la demencia y es entonces cuando se toma la decisión de intervenir mediante la alimentación artificial mediante una sonda enteral.

Durante muchos años la alimentación e hidratación mediante sonda nasogástrica (SNG) se ha considerado el tratamiento de elección. Algunas revisiones anteriores al $2000^{1}$, ponen en entredicho parte de los objetivos que justificaban la indicación de colocar una sonda en enfermos con demencia avanzada: no impide las neumonías por aspiración ni las consecuencias de la desnutrición, no mejora la supervivencia ni impide ni corrige las úlceras por presión, no reduce el riesgo de otras infecciones, no mejora el estado funcional del enfermo ni tampoco su bienestar. Dichos estudios consideran que la alimentación oral del enfermo con demencia, aun en pequeñas cantidades, es suficiente, más cómoda y sus resultados no son peores que con la alimentación por sonda.

No debemos olvidar que la alimentación tiene un valor social profundo. De hecho se le concede un papel trascendental en la recuperación de la enfermedad en nuestra sociedad. Desde otro punto de vista, algunos autores sostienen que la nutrición y la alimentación artificial son formas de tratamiento médico y que por dicho motivo, deben estar sujetas a los mismos criterios de análisis de cargas y beneficios que el resto de los tratamientos para su indicación'. También hay que tener en cuenta que la colocación de una sonda de gastrostomía (PEG), se realiza a través de un procedimiento quirúrgico invasivo con riesgo significativo de eventos adversos postoperatorios ${ }^{1}$.

El debate no está resuelto y solamente el análisis objetivo de las cargas y beneficios junto a los deseos del enfermo manifestados previamente o tratando de averiguar qué es lo que desearía si nos lo pudiera decir, así como la opinión de la familia, podrán ayudarnos a tomar la decisión ${ }^{1}$. A pesar de estas cuestiones y de los muchos documentos de trabajo que cuestionan la utilidad de la alimentación enteral en la demencia avanzada, ésta sigue siendo una intervención frecuente y es muy posible que, con el aumento esperado de las personas con demencia avanzada, la alimentación enteral se torne cada vez más común.

Por ello nos propusimos realizar una revisión sistemática de la literatura que diera respuestas a las siguientes preguntas según la evidencia disponible. ¿La alimentación por sonda PEG en pacientes con demencia avanzada previene las aspiraciones? ¿Previene la desnutrición y sus consecuencias? ¿Se aumenta la supervivencia? ¿Cumplen estos pacientes criterios de terminalidad? ¿Son subsidiarios de cuidados paliativos?

\section{MATERIAL Y MÉTODOS}

Se realizó una revisión sistemática de artículos publicados desde 2000 a 2012 en inglés y en español. Se incluyeron artículos de revisión, estudios descriptivos y estudios analíticos de cualquier contexto de la asistencia sanitaria (hospitales para enfermos agudos, centros geriátricos y residenciales y la comunidad), pero se excluyeron casos clínicos y artículos de ideología religiosa. Se realizaron búsquedas en las siguientes bases de datos: Pubmed, Google Scholar, Registro Especializado de Ensayos Controlados del Grupo Cochrane de Demencia y Trastornos Cognitivos (Cochrane Dementia and Cognitive Improvement Group). Se utilizaron los siguientes términos de búsqueda y con los operadores booleanos AND y OR: Dementia, Enteral nutrition, Nutritional support, Endoscopic gastrostomy, Tube feeding, Peg, Enteral feeding, Ethics, Quality of life, Palliative care, Dysphagia y Make decisions.

Se obtuvieron 96 artículos que cumplían los criterios descritos. 24 artículos en español y 72 artículos en inglés.

\section{RESULTADOS}

\section{1. ¿La alimentación a través de sonda PEG en pacientes con demencia severa previene las aspiraciones?}

Cuando aparece disfagia el equipo asistencial suele plantearse, entre otras alternativas, la colocación de una sonda de alimentación. El razonamiento básico para utilizarla es salvar el "obstáculo" orofaríngeo con una deglución más segura, mejorar la situación nutricional del paciente, evitar las neumonías por aspiración, prevenir la aparición de úlceras por presión, garantizando más confort 
y supervivencia al paciente. La colocación de una SNG o PEG, aun siendo una técnica sencilla, no está exenta de complicaciones ${ }^{2,3,6}$. En los últimos años ha aumentado progresivamente el número de pacientes con diagnóstico de demencia avanzada, portadores de un tubo para la alimentación como la alternativa que ofrece la ciencia para mejorar la calidad de vida y como prevención de las complicaciones de la disfagia ${ }^{5,14}$.

Una revisión publicada en la revista JAMA en 1999 por Finucane ${ }^{1}$, cuestionaba la efectividad de esta medida en los pacientes con demencia severa. Finucane demostraba que además de no cumplir las expectativas de mejorar el estado nutricional, prevenir aspiraciones, etc, producían efectos no deseados y complicaciones en cuando a la técnica y el manejo a corto, medio y largo plazo. Además de los riesgos que conlleva la técnica, Finucane añadía también el aumento de alteraciones conductuales que pueden conducir a la colocación de contenciones físicas. Tras la publicación de este trabajo, fueron muchos los estudios que plantearon una falta de evidencia en que la alimentación por sonda proporcionara beneficios al paciente con demencia avanzada.2,3,6,18.

Uno de esos trabajos fue la revisión sistemática de la literatura publicada por Sampson ${ }^{18}$. En ella la autora plantea que la decisión de usar hidratación y nutrición artificiales en una persona con demencia suele ser emotiva y compleja. Los familiares y cuidadores pueden solicitar la intervención porque están preocupados de que el paciente esté pasando hambre; los médicos pueden ser conscientes de los riesgos pero al sentirse presionados por distintos motivos: directivos institucionales, sociales e incluso legales, deciden intervenir.

En esta revisión de 452 artículos, la autora plantea que no hay pruebas definitivas de que la nutrición por sonda enteral sea eficaz para prolongar la supervivencia, mejorar la calidad de vida, lograr una mejor alimentación o reducir el riesgo de úlceras de decúbito. En realidad, puede aumentar el riesgo de desarrollar neumonía debido a la inhalación de pequeñas cantidades de alimentos e incluso provocar la muerte. Así pues plantea que este área resulta difícil de investigar pero que se requieren estudios mejor diseñados para aportar pruebas más consistentes. Gomez-Bustos, et.al ${ }^{19}$ plantean que los mayores beneficios para el enfermo se obtienen mediante estrategias destinadas a mejorar el estado de las piezas dentales, la posición durante la ingesta, la utilización de espesantes y la supresión de fármacos sedantes que interfieren la deglución.

La Sociedad Europea de Nutrición Enteral y Parenteral (ESPEN), publicó en $2005^{11}$ un artículo en el que establecía el algoritmo para la toma de decisiones de la vía de acceso óptima para la nutrición enteral en caso de vía oral no adecuada o imposible). (Gráfico 1). En dicho algoritmo vemos que la SNG y la sonda PEG no estarían indicadas en las situaciones que cursan con riesgo de broncoaspiración como sucede en las demencias.

Para estas situaciones, la ESPEN propone la sonda nasoyeyunal y la PEG en yeyuno como técnicas indicadas, sin embargo ambas técnicas no pueden colocarse de forma ambulatoria en el domicilio del paciente en el caso de salida accidental o recambio, ya que requieren control radiológico posterior y tampoco están exentas de complicaciones; con las sondas nasoyeyunales es habitual la aparición de diarreas y las complicaciones más frecuentes de las sondas PEG-yeyuno son la migración de las mismas a estómago y la obstrucción de la propia sonda al ser de calibre pequeño. Además que según la revisión de Sampson ${ }^{18}$ tampoco éstas prevenían las aspiraciones.

\section{2. ¿La alimentación a través de sonda PEG en pacientes con demencia severa previene la desnutrición y sus consecuencias?}

En las demencias la pérdida de peso está presente desde fases tempranas, aun cuando todavía no ha aparecido el problema deglutorio. GomezBustos ${ }^{19}$ plantea que en muchos pacientes en la que la enfermedad está avanzada, se observa una capacidad de deglución suficiente para no perder peso. Incluso con bajo peso corporal y demencia avanzada pueden sobrevivir por largos periodos de tiempo. También plantea que la preocupación por el estado nutricional del paciente con demencia no debe plantearse cuando aparece la disfagia, sino que hay que prestarle atención durante toda la evolución de la enfermedad, tomando en cada fase las medidas oportunas.

En muchos de los estudios se llega a la conclusión de que con las sondas de alimentación no se logra una mejoría en el estado nutricional o en las consecuencias delanutrición deficitaria $2,4,5,10,11,12,16,18$. La guía ESPEN ${ }^{11}$ no considera indicado el uso de sondas de alimentación en la última fase de la 
demencia. Tampoco se logra prevenir la aparición o mejorar el estado de las úlceras por presión secundarias al estado de malnutrición.

Desde un punto de vista práctico, en demencias evolucionadas podemos encontrarnos con enfermos que aceptan los alimentos pero que se atragantan y sufren aspiraciones pulmonares, y otros que rechazan el alimento o no hacen ningún acto de deglución. Podemos pensar que los primeros sentirán hambre y, en este sentido, decir que sufren si no se les alimenta. Sin embargo, la alimentación artificial mediante sondas requiere su aceptación por parte del enfermo, que no se encuentra en condiciones de comprender. En muchas ocasiones, para su mantenimiento se precisa del uso de contenciones físicas o químicas que empeoran su limitada calidad de vida. En cuanto a los segundos, podemos considerar el rechazo o la evitación de los alimentos como posibles deseos del enfermo, aun cuando no son enfermos competentes. En ambos supuestos el uso de medidas artificiales de nutrición y de hidratación es desproporcionado. Una adecuada información puede aliviar los sentimientos de culpa que surgen en los familiares cuando se retira la nutrición e hidratación en estos enfermos y, por otro lado se les puede animar a proporcionar alimentos por la boca como una forma de cuidado básica, con la ventaja de permitir que los familiares satisfagan sus preocupaciones emocionales, éticas y psicológicas ${ }^{4,19}$.

\section{3. ¿La alimentación a través de sonda PEG en pacientes con demencia severa aumenta la supervivencia?}

El primer estudio empírico que demostró que la mortalidad en los pacientes con demencia alimentados mediante PEG es elevada fue en el año $2000^{21}$. En él se describen cifras de mortalidad inicial del $28 \%$ en los primeros 30 días tras la colocación de la sonda PEG. Otros estudios describen que tras 6 meses, sólo el $50 \%$ de los pacientes con demencia avanzada es probable que sobrevivan, mientras que otros demuestran un $54 \%$ de mortalidad a 30 días en pacientes con demencia y tras un año del $70-90 \% \%^{3,4,5,7,15}$.

En los anteriores estudios se recomienda un periodo de enfriamiento o de gracia de 30 a 60 días durante los que se recomienda la alimentación por SNG y si sobreviven valorar de nuevo la sonda PEG.

$\mathrm{Se}$ han descrito como factores que afectan negativamente a la supervivencia en estos pacientes la desnutrición, hipoalbuminemia previa a la colocación de la PEG, edad avanzada (algunos autores hablan de mayores de 70 años y otros autores de 80 años), existencia de comorbilidad medida por el índice de Charlson (mortalidad tardía), proteína $\mathrm{C}$ reactiva (mortalidad temprana), deterioro cognitivo, demencia avanzada, presencia de úlceras por presión, gastrectomizados, patología cardiaca, diabetes, varones, e índice de Karnofsky $>50^{3,5,7,8,9,9,15,17,21}$.

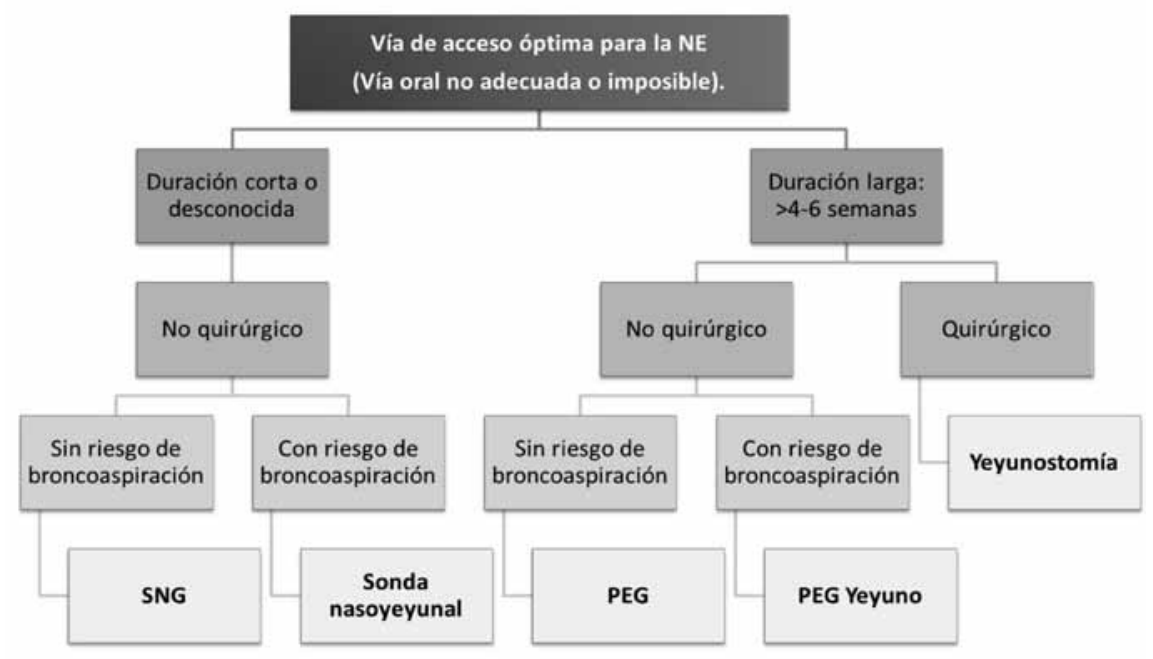

Gráfico 1:

ESPEN guidelines on artificial enteral nutrition-Percutaneous endoscopic gastrostomy (PEG).

Clinical Nutrition 2005. 


\section{4. ¿Los pacientes con demencia severa, se tratan de forma paliativa?}

No deja de ser sorprendente que a pesar de la falta de evidencia sobre su beneficio, el número de pacientes portadores de sondas de alimentación en las demencias avanzadas es muy elevado. En este sentido, algunos autores ${ }^{19}$ plantean como responsables factores económicos, ya que la preparación de las comidas para estos pacientes conlleva trabajo, el tiempo de administración es más largo y se requiere más personal. Todo ello encarece el cuidado en las instituciones si se compara con una alimentación enteral por sonda, y por ello estos autores llegan a la conclusión que el sistema sanitario favorece de alguna manera este tipo de nutrición. Otros autores ${ }^{9}$ hablan de factores sociales, creencias personales, etc.

En cuanto a la ingesta de alimentos, se piensa que el consumo insuficiente de alimentos provoca hambre y sed angustiantes. Los datos sugieren que entre los pacientes cognitivamente intactos, el rechazo de alimentos y agua en el contexto de una enfermedad terminal no es doloroso ${ }^{10}$. Algunos estudios indican que la alimentación enteral puede tener un efecto opuesto al deseado y, en realidad, aumentar la mortalidad, la morbilidad y reducir la calidad de vida, puede empeorar la incontinencia urinaria y fecal que se asocia con un mayor riesgo de úlceras de decúbito y puede aumentar las secreciones pulmonares ${ }^{10,12}$.

La demencia no es asumida como una enfermedad terminal, aunque ya en 1996 la National Hospice Association estableció los criterios que un paciente con demencia avanzada tendría que cumplir para ser subsidiario de cuidados paliativos ${ }^{22}$. La demencia desde siempre se ha considerado como "cosa de la edad", o como una enfermedad de curso prolongado en el tiempo que hace difícil asumir que ha llegado a un estadio final. Todo ello provoca una ambivalencia en la toma de decisiones entre los familiares, que como bien plantea Lubart ${ }^{9}$, retirar una sonda es una decisión más difícil que implantarla.

Tal y como plantea Gómez-Bustos ${ }^{19}$, el momento de la decisión de colocar una sonda de alimentación se ve influido por las propias creencias y convicciones de los cuidadores, por la comprensión y actitud médica y el modelo de fin de vida que se plantee a la familia. Se suele hacer una lectura únicamente tecnológica de la enfermedad: salvar un obstáculo para que llegue el nutriente al estómago sin tener en cuenta al individuo en todas sus dimensiones.
Algunos autores ${ }^{8,20}$, realizan un análisis de la aplicación de los principios de bioética en la demencia avanzada; destacando la importancia de saber dar una información clara y exhaustiva a las familias para que la toma de decisiones difíciles sea lo más cercana a los deseos del paciente.

La formación en cuidados paliativos y más concretamente en los cuidados paliativos no oncológicos, es muy escasa. Este hecho favorece que la fase final en la demencia no se reconozca fácilmente ${ }^{10}$ y nos sea más sencillo informar que la solución, o como dice Chernoff ${ }^{13}$ la "alternativa tecnológica" a ofrecer, es una sonda de alimentación y se deja en manos de la familia una decisión que además de no ser correcta en la técnica como se ha indicado anteriormente, debería contemplar la situación global del individuo, la dignidad, el confort del paciente, las dudas de los familiares y además debería respetar las voluntades del paciente, lo que no siempre sucede.

Determinados profesionales defienden la instauración de métodos artificiales debido al esfuerzo que supone dar la comida en estas condiciones; sin embargo, este argumento valora más la conveniencia de los cuidadores que el interés del enfermo ${ }^{16}$

También es cierto que los responsables de los enfermos alojados en residencias asistidas pueden mostrarse reticentes a negar o retirar la nutrición y la alimentación si no existe una declaración explícita del paciente en un documento de voluntades anticipadas (en España adquirió estatus legal con la publicación de la Ley 41/2002) ${ }^{12}$.

También hay cuestiones respecto de si los cuidadores informales que dan su consentimiento están debidamente informados tanto de los beneficios como de los daños potenciales de la intervención y de las alternativas para optimizar la calidad de vida ${ }^{8,20,23}$.

\section{DISCUSIÓN}

La alimentación tiene un valor social profundo y la decisión de comenzar la alimentación enteral es polémica y está influenciada por cuestiones éticas complejas. La decisión de intervenir difiere con la necesidad clínica, la práctica local y la preferencia de médicos y cuidadores. Las justificaciones frecuentes proporcionadas pueden incluir la prolongación de la vida mediante la corrección de la malnutrición, la reducción del riesgo de aspiración y úlceras de decúbito, neumonía y otras 
infecciones o la optimización de la calidad de vida al promover la comodidad física.

Sin embargo, no se ha establecido si con la alimentación enteral se logra alguno de estos resultados y se puede, en cambio, provocar daño. Algunos estudios indican que la alimentación enteral puede tener un efecto opuesto al deseado $y$, en realidad, aumentar la mortalidad, la morbilidad y reducir la calidad de vida.

La PEG es un procedimiento quirúrgico invasivo con riesgo significativo de eventos adversos postoperatorios y numerosos estudios plantean factores que pueden afectar negativamente a la supervivencia. Puede provocar agitación que lleve al paciente a requerir contención física o química para evitar la autoextubación y esto puede ser visto como una violación del derecho a dignidad de los pacientes. También hay cuestiones respecto de si los cuidadores informales que dan su consentimiento están debidamente informados tanto de los beneficios como de los daños potenciales de la intervención y de las alternativas para optimizar la calidad de vida.

A pesar de estas cuestiones y de los muchos documentos de trabajos que cuestionan la utilidad de la alimentación enteral en la demencia avanzada, ésta sigue siendo una intervención frecuente. $Y$ es posible que, con el aumento de las personas con demencia avanzada, la alimentación enteral se torne cada vez más común.

\section{BIBLIOGRAFÍA}

1. Finucane $T$, Christmas $C$, Travis $K$. Tube feeding in patients with advanced dementia: A review of the evidence. JAMA 1999; 282(14):1.365-70.

2. Gillick MR. Rethinking the role of tube feeding in patients with advanced dementia. NEJM 2000:342:206-10.

3. Meier DE, Ahronheim JC, Morris J, Baskin-Lyons S, Morrison RS. High short-term mortality in hospitalized patients with advanced dementia: Lack of benefit of tube feeding. Archives of Internal Medicine 2001;161:594-59.

4. Young K. To feed or not to feed: tube feeding in patients with Advanced Dementia. Nutrition Reviews. Volume 59, Issue 3, pages 86-88, March 2001.

5. Skelly $\mathrm{RH}$. Are we using percutaneous endoscopic gastrostomy appropriately in the elderly? Curr Opin Clin Nutr Metb Care. 2002;5:35-42.

6. Li I. Feeding tubes in patients with severe dementia. Am Fam Physician. 2002 Apr 15;65(8):1605-10, 1515.

7. Murphy LM, Lipman TO. Percutaneous endoscopic gastrostomy does not prolong survival in patients with dementia. Archives of Internal Medicine 2003;163:1351-3.

8. Valero Zanuy M.A, Alvarez Nido R, de Diego Muñoz R, Moreno Villares J.M, León Sanz M. ¿Se debe limitar el esfuerzo terapéutico en nutrición artificial?. Rev Clin Esp 2003;203(12):582-8.

9. Lubart E, Leibovitz A, Habot B. Attitudes of relatives and nursing staff toward tuboenteral feeding in severely demented patients. Am J Alzheimers Dis Other Demen. 2004;19:31-4.

10. Monteleoni C, Clark E. Using rapid-cycle quality improvement methodology to reduce feeding tubes in patients with advanced dementia: Before and after study. BMJ. 2004;329:491-4.

11. Löser Ch, Aschl G, Hébuterne C, et.al. ESPEN guidelines on artificial enteral nutrition- Percutaneous endoscopic gastrostomy (PEG). Clinical Nutrition (2005) 24, 848-861.

12. Carro-García T. Soporte nutricional en pacientes con demencia avanzada. Rev Esp Geriatr Gerontol. 2006;41:734. - vol.41 núm 01.

13. Chernoff R. Tube feeding patients with dementia. Nutr Clin Pract 2006;21:142-6.

14. Cervo FA, Bryan L, Farber S. To PEG or not to PEG: A review of evidence for placing feeding tubes in advanced dementia and the decision-making process. Geriatrics. 2006:61:30-5.

15. Delegge MH. Percutaneous endoscopic gastrostomy in the dementia patient: Helpful of hindering? Am J Gastroenterol. 2008;103:1018-20.

16. Delegge $\mathrm{MH}$. Tube feeding in patients with dementia: Where are we?. Nutr Clin Prac. 2009;24:214-6.

17. Gillick MR, Volandes AE. The standard of caring why do we still us feeding tubes in patients with advanced dementia?. JAMDA Volume 9, Issue 5 , Pages 364-367, junio de 2008.

18. Elizabeth L Sampson, Bridget Candy, Louise Jones. Alimentación enteral para pacientes mayores con demencia avanzada (Revision Cochrane traducida). Biblioteca Cochrane Plus 2009 Número 3. Oxford: Update Software Ltd. Disponible en: http://www.update-software. com. (Traducida de The Cochrane Library, 2009 Issue 2 Art no. CD007209. Chichester, UK: John Wiley \& Sons, Ltd.).

19. Gómez Busto F, Andía V, Ruiz de Alegría L, Francés I. Abordaje de la disfagia en la demencia avanzada. Rev. Esp Geriatr Gerontol. 2009; 44(S2):29-36.

20. Gisbert Aguilar A. Aspectos éticos de la alimentación e hidratación en pacientes con deterioro cognitivo severo. Med. Pal Vol. 16: N. ${ }^{\circ} 2 ; 100-110,2009$.

21. Sanders DS, Carter MJ, D'Silva J, James G, Bolton RP, Bardhan KD. Survival analysis in percutaneous endoscopic gastrostomy feeding: a worse outcome in patients with dementia. Am J Gastroenterol. 2000 Jun;95(6):1472-5.

22. Arriola Manchola E, González Larreina R, Ibarzabal Aramberri R, Buiza Bueno C. Criterios para la inclusión de un paciente con síndrome demencial en la fase asistencial de cuidados paliativos. Revista española de Geriatría y Gerontología. 2002 Jul;37(4).

23. Formiga F, Vidaller A, Mascaró J, Pujol R. Morir en el hospital por demencia en fase terminal: análisis de la toma de decisiones después de un programa educativo. Rev Esp Geriatr Gerontol. 2005;40(1):18-21.

Agradecimientos: A Francisco José Ortiz González y Urbano Muñoz por su ayuda con la traducción de los textos. 